\title{
Analysis of Navigation Error of an Integrated GNSS-Vision System Based on Geometric Allocation of Landmarks via Modelling of the Urban Environment
}

\author{
Chi-Ho Park and Nam-Hyeok Kim
}

\begin{abstract}
In this paper, we propose a reliable, precise and stable technique for solving common positioning problems, such as the signal occlusion of a navigation satellite in an urban canyon, and increasing positioning error due to a limited number of available navigation satellites. Generally, satellite navigation positioning systems cannot calculate a position coordinate when its satellite signal is occluded by some obstacle, and for this reason the navigation satellites system cannot be used for a variety of positioning applications. As a solution we propose a fusion system of the navigation satellites system and a vision system that supplements the weakness of the navigation satellites system with a vision system. In this method, some target objects that have accurate position coordinates are installed into the vision system, for example, in an outdoor shaded area like an urban canyon. When the vision system recognizes a target object it loads the accurate coordinate of that target object. Then, it measures the distance by using the disparity from the camera sensor to the target object. These distance and object coordinate data are used for positioning with the navigation satellites system's data. This fusion system can be a solution for outdoor shaded areas, which now account for $47 \%$ of the earth. And, it can reduce position errors that result from the limited number of available satellites, and the multipath effect.
\end{abstract}

Index Terms - GNSS, vision, positioning.

\section{INTRODUCTION}

The Global Positioning System (GPS) was developed in the United States for military purposes. However, since the 1990s, after being opened to the private sector, it has become widely used for vehicle and aircraft navigation, communications, science, agriculture, and exploration. In addition, the Soviet Union's navigation satellite system GLONASS was also opened to the private sector and this has allowed the Global Navigation Satellite System (GNSS) to be utilized for more purposes, and there have been many recent studies involving GNSS applications [1]-[4].

Today's navigation satellite system provides location information services within around $100 \mathrm{~m}$ (drms) accuracy at any time, regardless of the number of users. The system calculates a three-dimensional position by using triangulation, so it can be operated whenever the receiver can take signals from more than 4 satellites. However, locations within a city or in mountainous areas cannot always receive satellite

Manuscript received June 9, 2015; revised December 4, 2015.

The authors are with the Daegu Gyeongbuk Institute of Science \& Technology, Korea (e-mail : chpark@dgist.ac.kr, nhkim@dgist.ac.kr). signals because signals may be blocked by high buildings or mountains. In such cases, the position error will be bigger or shaded areas (without coverage) will occur. Consequently, to solve these problems, many researchers have proposed techniques using the navigation satellite system and other applications [5], [6].

One representative type of such approaches is an integration system involving the navigation satellite system and an Inertial Measurement Unit (IMU). This system is a device used by submarines, aircraft, missiles, etc. of various countries to detect their own location and to drive to a targeted destination. The operating principle involves calculating the moving variance by using accelerometers, after determining cardinal points by gyroscope. The moving object can always calculate its current position and velocity after being input with the initial position. The advantage of IMU is that it is not affected by weather or jamming. However, when moving over a long distance, errors are accumulated. So, GPS correction is needed. In the case that signals are not received from satellites, the errors of the IMU exponentially increase with distance. Therefore, factors which cause shaded areas and obstacles limit the uses of kinematic positioning [7].

Due to rapid industrialization and the increasing growth of urban canyons, the areas and environments where the navigation satellite system can be used is being reduced. Fig. 1 shows a general situation for navigation satellite system signal reception in an urban canyon.

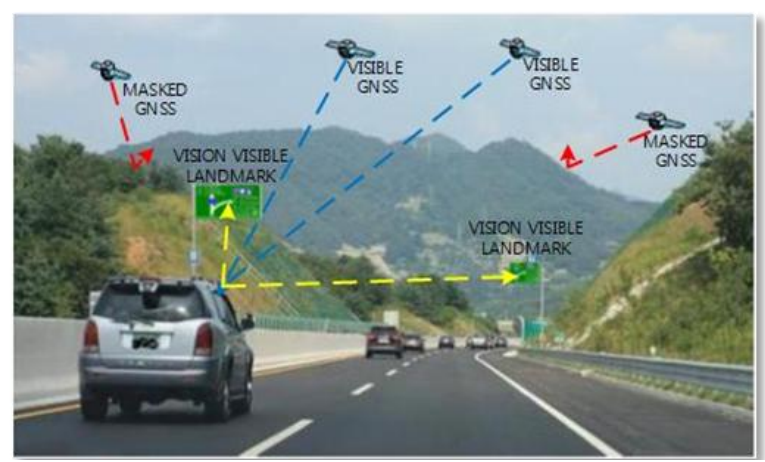

Fig. 1. The situation for signal reception from the navigation satellite in an urban canyon.

A vision based navigation system was recently reported at a DARPA unmanned vehicle conference in the United States which, in part, dealt with obstacle recognition and detection [8], [9]. In particular, one team reported that they had used only a vision system for detection and recognition. But the vision system had some constraints, such as a real time processing problem due to the large amounts of data, and a 
recognition error due to external lighting changes.

Other recent studies using vision systems have reported significant progress by improving the computer's performance and data processing time, through the development of integration technology and the development of a broadband camera [10], [11].

The advantages of a vision system are its wide range and long detection distance, the ease of data processing due to its similarity with the human visual system, and the provision of a variety of information. A stereo vision system, which is capable of solving many of the problems of a monocular vision system, uses a stereo matching algorithm for extracting a depth map, and an obstacle detection algorithm based on this depth map [12].

In this paper, we propose a method to integrate both the navigation satellite system and the vision system using a weights decision analysis for precise positioning.

\section{ALGORITHM}

\section{A. Positioning Using a Navigation Satellite System}

Usually, a position is obtained by using a receiver chip in point positioning mode, which is mounted on the vehicle and receives the L1 C/A (Coarse/Acquisition) code from the navigation satellites. The $\mathrm{C} / \mathrm{A}$ code observation equation for the navigation satellites system is given as follows.

$$
\begin{aligned}
& P_{i, 1}^{k}=\rho_{i}^{k}+T_{i}^{k}+\frac{I_{i}^{k}}{f_{1}^{2}}+c\left(d t_{i}-d t^{k}\right)+e_{i, 1}^{k} \\
& \rho_{i}^{k}=\sqrt{\left(x^{k}-x_{i}\right)^{2}+\left(y^{k}-y_{i}\right)^{2}+\left(z^{k}-z_{i}\right)^{2}}
\end{aligned}
$$

where, $i$ and denote receiver and satellite, respectively.

$P_{i, 1}^{k}: \mathrm{L} 1 \mathrm{C} / \mathrm{A}$ code pseudorange between the receiver and the satellite $(\mathrm{m})$;

$\rho_{i}^{k}$ : actual geometric distance between receiver and satellite $(\mathrm{m})$;

$T_{i}^{k}:$ tropospheric delay error $(\mathrm{m}) ;$

$\frac{I_{i}^{k}}{f_{1}^{2}}$ : ionosphereric delay error $(\mathrm{m})$;

$c:$ speed of light $(\mathrm{m} / \mathrm{s})$;

$d t_{i}:$ receiver clock error $(\mathrm{sec})$;

$d t^{k}$ : satellite clock error (sec);

$e_{i, 1}^{k}$ : measurement error.

Ionospheric delay effects and satellite clock errors are removed by a navigation message from the satellites. Tropospheric delay effects are removed by models that account for the dry and wet refractivity at the surface of the Earth. Multipath error is not assumed. Inter-frequency bias is ignored because of its small value. As a consequence, (2) can be used for the observation equation to compute the receiver's position in 3-dimensional space.

$$
P G_{i, 1}^{k}-T_{i}^{k}-\frac{I_{i}^{k}}{f_{1}^{2}}+c d t^{k}=\rho_{i}^{k}+c d t_{i}+e_{i, 1}^{k}
$$

We denote by $P_{i, c}^{k}$ in the left side of (2) and linearize (2) because it is a non-linear equation. After that, the Gauss-Markov Model (GMM) [13] is applied. The result is (3). The satellites' position coordinates are determined by using the navigation message. Unknown factors are the 3-dimensional position and receiver clock error.

$$
y=A \xi+e, \quad e \sim\left(0, \sigma_{0}^{2} P^{-1}\right)
$$

Each item is shown below; $\dot{\rho}$ is calculated by the receiver's initial position $\left(\dot{x}_{i}, \dot{y}_{i}, \dot{z}_{i}\right)$.

$$
y=\left[\begin{array}{ccc}
P_{i, 0}^{k} & - & P_{i, c}^{k} \\
P_{i, 0}^{l} & - & P_{i, c}^{l} \\
& \vdots & \\
P_{i, 0}^{q} & & P_{i, c}^{q}
\end{array}\right]: \text { Observation vector }
$$$$
A=\left[\begin{array}{cc}
-\frac{x^{k}-\dot{x}_{i}}{\dot{\rho}_{i}^{k}}-\frac{y^{k}-\dot{y}_{i}}{\dot{\rho}_{i}^{k}}-\frac{z^{k}-\dot{z}_{i}}{\dot{\rho}_{i}^{k}} & c \\
-\frac{x^{l}-\dot{x}_{i}}{\dot{\rho}_{i}^{l}}-\frac{y^{l}-\dot{y}_{i}}{\dot{\rho}_{i}^{l}}-\frac{z^{l}-\dot{z}_{i}}{\dot{\rho}_{i}^{l}} & c \\
\vdots & \\
-\frac{x^{q}-\dot{x}_{i}}{\dot{\rho}_{i}^{q}}-\frac{y^{q}-\dot{y}_{i}}{\dot{\rho}_{i}^{q}}-\frac{z^{q}-\dot{z}_{i}}{\dot{\rho}_{i}^{q}} & c
\end{array}\right]: \text { Design matrix }
$$$$
\xi=\left[\begin{array}{c}
\Delta x_{i} \\
\Delta y_{i} \\
\Delta z_{i} \\
d t_{i}
\end{array}\right]: \text { Unknown parameter vector }
$$

$e=\left[\begin{array}{c}e_{i}^{k} \\ e_{i}^{l} \\ \vdots \\ e_{i}^{q}\end{array}\right]:$ Measurement error vector

$n \times 1$

The unknown that is calculated in (3) is the increment with respect to the initial value.

$$
\hat{\xi}=\underbrace{\left(A^{T} P A\right)^{-1}}_{N} A^{T} P y
$$

The increment from (4) is added to the receiver's initial position and then the receiver's position is updated. This process is iterated until the increment is under the particular threshold value. After this process, the receiver's position is determined.

$$
\left[\begin{array}{c}
x_{i} \\
y_{i} \\
z_{i}
\end{array}\right]_{\text {update }}=\left[\begin{array}{c}
x_{i} \\
y_{i} \\
z_{i}
\end{array}\right]_{\text {initial }}+\left[\begin{array}{c}
\Delta x_{i} \\
\Delta y_{i} \\
\Delta z_{i}
\end{array}\right]
$$

The variance component can be computed by using (6). Also, the variance-covariance matrix for the estimates can be obtained using (7).

$$
\hat{\sigma}_{0}^{2}=\frac{\widetilde{e}^{T} P \widetilde{e}}{n-r k A}
$$


where, $\tilde{e}=y-A \hat{\xi}, n$ is the number of observations.

$$
D\{\hat{\xi}\}=\sigma_{0}^{2} N^{-1}
$$

\section{B. Fusion Positioning Equations}

The vision system obtains observation values by recognizing objects. This means that the sizes of the observation vector and design matrix get larger as the number of observations increases. The distance from the receiver to the target object can be computed by using the vision system, and the corresponding observation equation is as follows.

$$
\begin{aligned}
& P V_{i}^{a}=\rho_{i}^{a}+e_{i}^{a} \\
& \rho_{i}^{a}=\sqrt{\left(x^{a}-x_{i}\right)^{2}+\left(y^{a}-y_{i}\right)^{2}+\left(z^{a}-z_{i}\right)^{2}}
\end{aligned}
$$

$P V_{i}^{a}$ : distance estimated by the vision system from the specific object to the receiver $(\mathrm{m})$.

$\rho_{i}^{a}$ : actual distance from the specific object to the receiver.

$x^{a}, y^{a}, z^{a}:$ three-dimensional position of a specific object.

$x_{i}, y_{i}, z_{i}:$ three-dimensional position of the receiver.

After linearization, (9) can be rewritten as follows.

$$
z_{0}=K \xi+e_{0}, \quad e_{0} \sim\left(\sigma_{0}^{2} P_{0}^{-1}\right)
$$

$Z_{0}=\underbrace{\left[P V_{i}^{a}-\rho_{i}^{a}\right]}_{1 \times 1}:$ Observation vector

$A=\left[-\frac{x^{a}-\dot{x}_{i}}{\dot{\rho}_{i}^{a}}-\frac{y^{a}-\dot{y}_{i}}{\dot{\rho}_{i}^{a}}-\frac{z^{a}-\dot{z}_{i}}{\dot{\rho}_{i}^{a}} \quad 0\right]:$ Design matrix

$\xi=\left[\begin{array}{c}\Delta x_{i} \\ \Delta y_{i} \\ \Delta z_{i} \\ d t_{i}\end{array}\right]:$ Unknown parameter vector

The distance measurement from the vision system can be used as an additional observation and then the Gauss-Markov adjustment model with stochastic constraints is applied as shown in (10).

$$
\hat{\xi}=\left(N+K^{T} P_{0} K\right)^{-1}\left(c+K^{T} P_{0} z_{0}\right)
$$

The residual of the distance estimated by the vision system is $\tilde{e}_{0}=Z_{0}-K \hat{\xi}$ and the estimated variance component is (11).

$$
\hat{\sigma}_{0}^{2}=\frac{\tilde{e}^{T} P \tilde{e}+\tilde{e}_{0}^{T} P_{0} \tilde{e}_{0}}{n-m+l}
$$

Here, $n$ is the observed number of navigation satellites, $m$ is the number of unknown parameters (coordinates 3, receiver's clock error 1), $l$ is the number of the distance measurement obtained from the vision system. Also, the variance-covariance matrix for the estimates can be computed by using (12).

$$
D\{\hat{\xi}\}=\hat{\sigma}_{0}^{2}\left(N+K^{T} P_{0} K\right)^{-1}
$$

where, $a$ is the specific object, $i$ is a receiver, and each of the items are as follow.

$P V_{i}^{a}$ : distance estimated by the vision system from the specific object to the receiver $(\mathrm{m})$.

$\rho_{i}^{a}$ : actual distance from the specific object to the receiver.

$x^{a}, y^{a}, z^{a}:$ three-dimensional position of a specific object.

$x_{i}, y_{i}, z_{i}:$ three-dimensional position of the receiver.

\section{PERFORMANCE ANALYSIS}

Experiments were conducted to evaluate the reliability and stability of positioning based on the integration of the navigation satellites system and the vision system. The receiver of the navigation satellite system was the DL-V3 Real Time Kinematic (RTK) from Novatel, for the base station and the rover. The antenna was GPS-702-GGL from Novatel, the RF modem was the PDL rover kit of $450 \mathrm{MHz}$, and the Inertial Measurement Unit (IMU) was CG-5100 from $\mathrm{KVH}$. Also, a stereo camera was used as the image sensor.

The focal length of the image sensor was $12 \mathrm{~mm}$ and the baseline of the stereo camera ( $x$ coordinate difference between the two cameras) was $300 \mathrm{~mm}$.

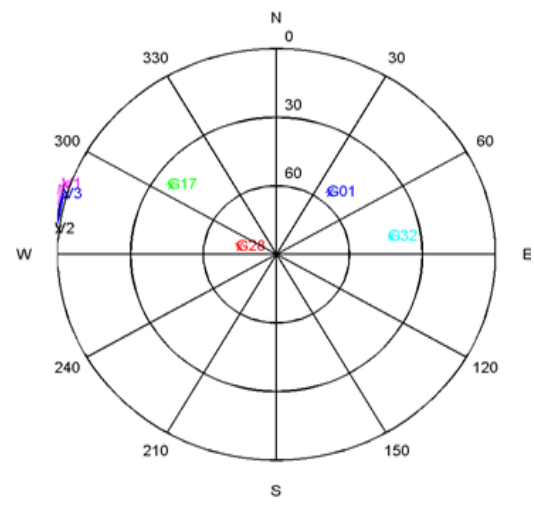

Fig. 2. Sky plot of the static state when the DOP was not considered.

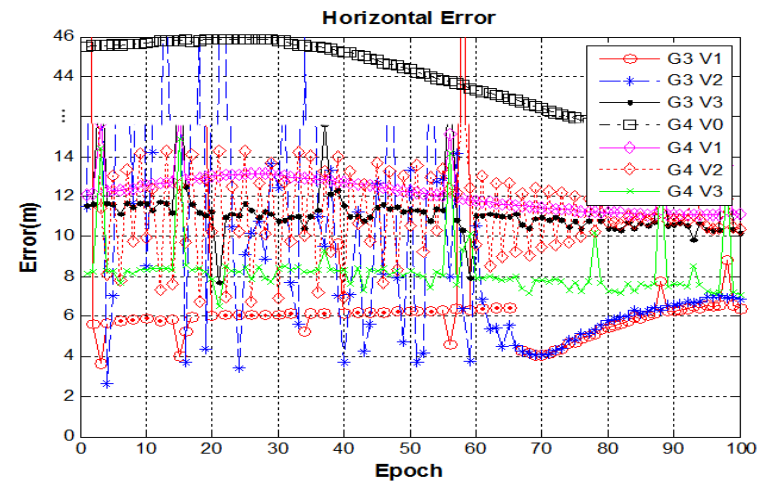

Fig. 3. Horizontal Position Error for static state when the DOP was not considered.

We compared the static and kinematic states of a vehicle in the experiments. Also we compared situations where the DOP was, and was not, considered. The following are the results of 
experiments in the static state. Fig. 2 shows the sky plot of the static state when the DOP was not considered.

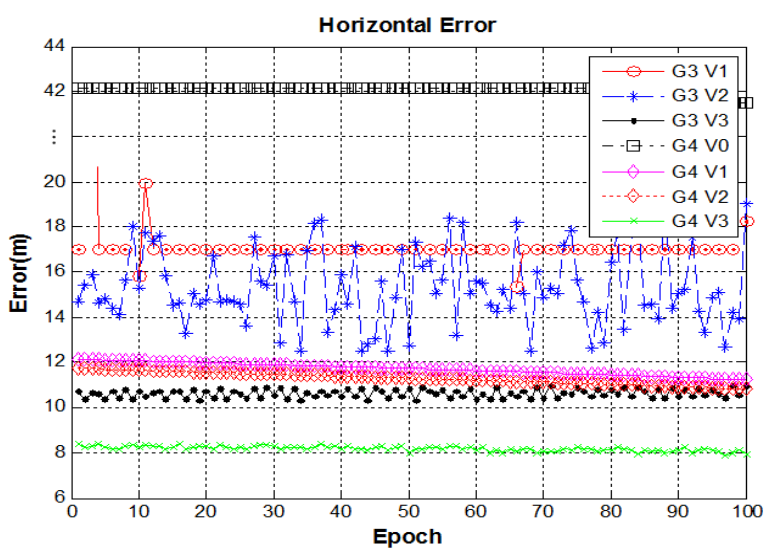

Fig. 4. Horizontal Position Error of the simulations when the DOP was not considered

Fig. 3 and Fig. 4 show the Horizontal Positioning Error for static and simulation states when the DOP was not considered. (In the Figures, $\mathrm{G}$ means the number of visible navigation satellites and $\mathrm{V}$ means the number of target objects for the vision system. For example, G3V1 means that there are 3 navigation satellites and one vision target object).

Fig. 5 shows the sky plot of the kinematic state when the DOP was not considered.

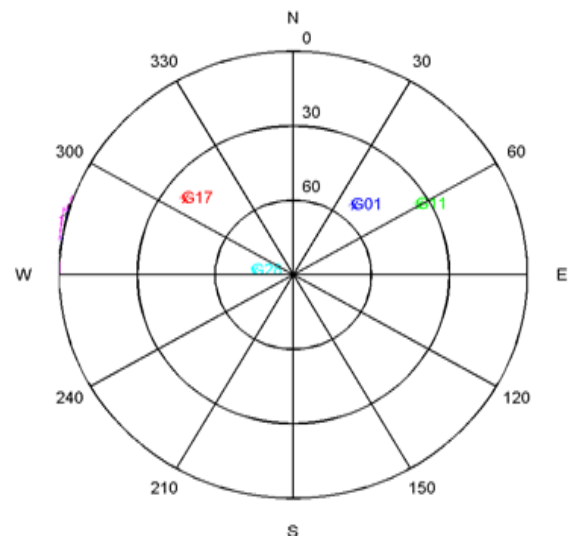

Fig. 5. Sky plot of the kinematic state when the DOP was not considered.

Fig. 6 and Fig. 7 show the Horizontal Positioning Error for kinematic and simulation states when the DOP was not considered.

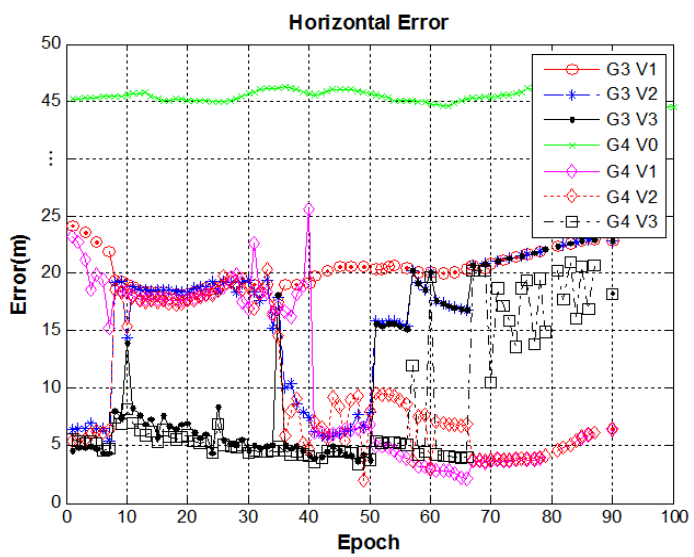

Fig. 6. Horizontal Position Error for kinematic state when the DOP was not considered

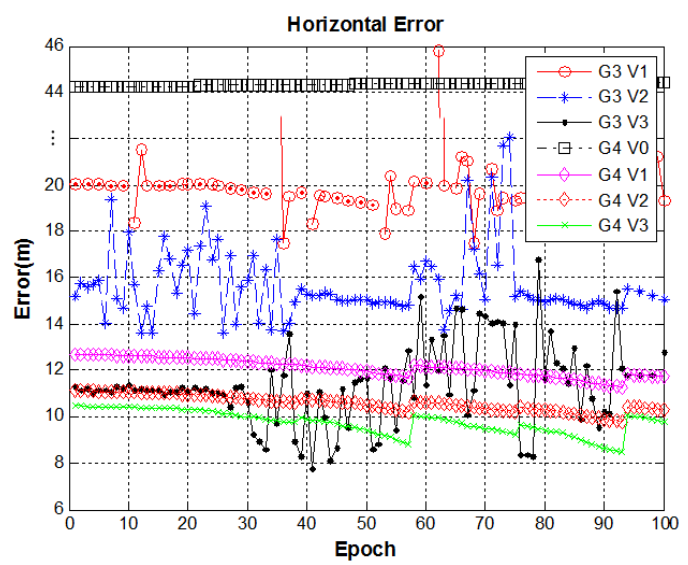

Fig. 7. Horizontal Position Error of the simulations when the DOP was not considered.

Fig. 8 shows the sky plot of the static state when the DOP was considered.

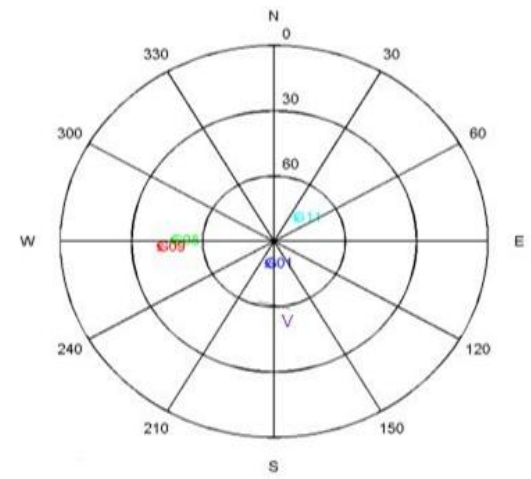

Fig. 8. Sky plot of the static state when the DOP was considered.

Fig. 9 and Fig. 10 show the Horizontal Positioning Error for static and simulation states when the DOP was considered.

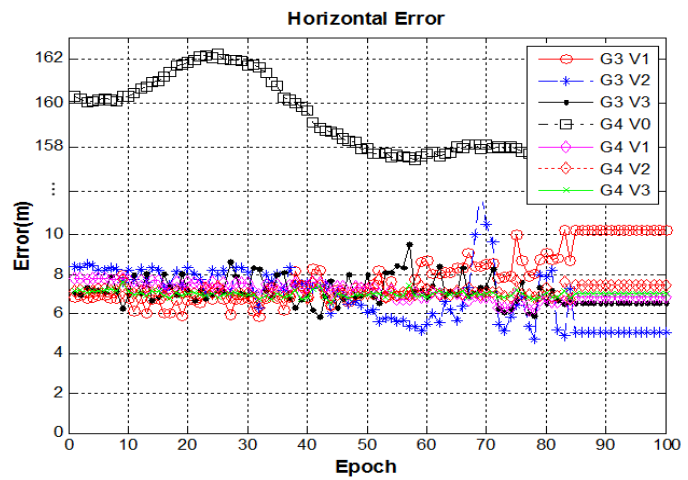

Fig. 9. Horizontal Position Error for static state when the DOP was considered.

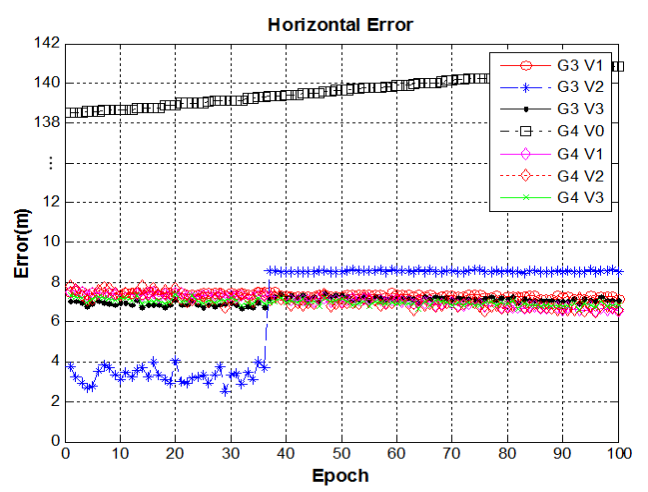

Fig. 10. Horizontal Position Error of the Simulations when the DOP was considered. 
Fig. 11 shows the sky plot of the kinematic state when the DOP was considered.

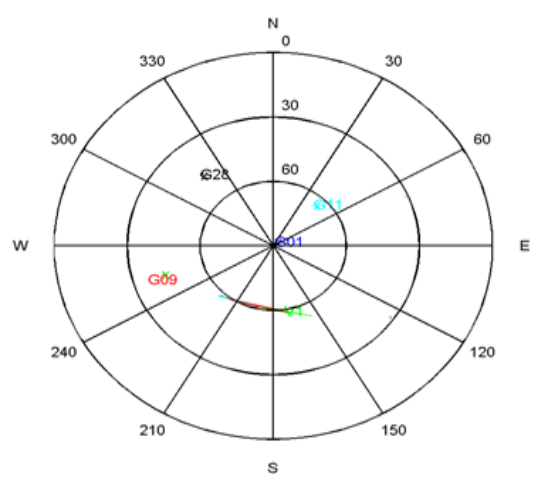

Fig. 11. Sky plot of the kinematic state when the DOP was considered.

Fig. 12 and Fig. 13 show the Horizontal Positioning Error for kinematic and simulation states when the DOP was considered.

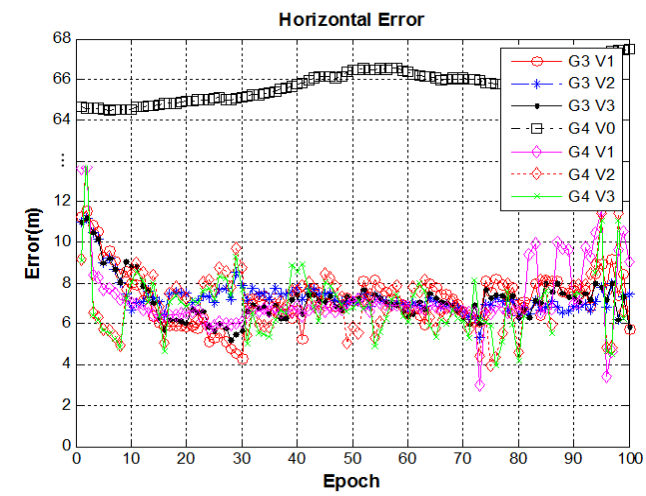

Fig. 12. Horizontal Position Error for static kinematic when the DOP was considered.

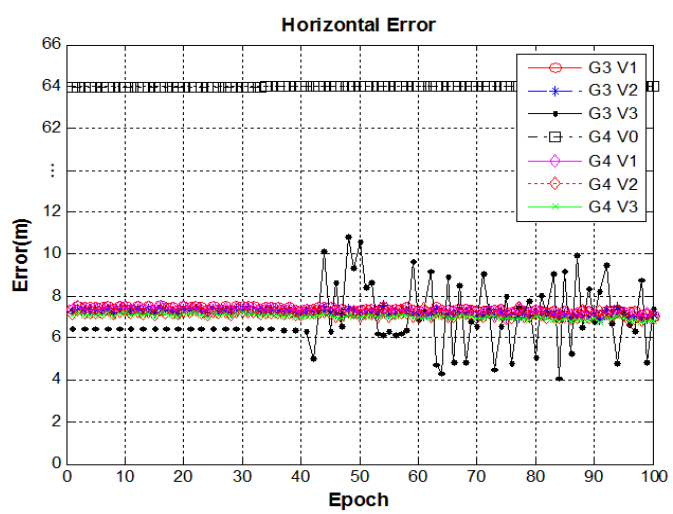

Fig. 13. Horizontal Position Error of the simulations when the DOP was considered.

\section{CONCLUSION}

Fig. 3 to Fig. 13 show the Horizontal Positioning Error according to variations in the number of satellites and vision's target objects.

The experimental results shown in Fig. 3 indicate that the RMS of Horizontal Position Error was $44.02 \mathrm{~m}$ when using only the satellite system. However G3V1 is $18.91 \mathrm{~m}, \mathrm{G} 3 \mathrm{~V} 2$ is $16.52 \mathrm{~m}, \mathrm{G} 3 \mathrm{~V} 3$ is $11.58 \mathrm{~m}, \mathrm{G} 4 \mathrm{~V} 1$ is $12.31 \mathrm{~m}, \mathrm{G} 4 \mathrm{~V} 2$ is $11.69 \mathrm{~m}$, and G4V3 is $8.45 \mathrm{~m}$. The performance of the Horizontal Position Error was improved by 57.04\%, 62.62\%, 73.69\%, $72.03 \%, 73.44 \%$, and $80.80 \%$.

The experimental results of Fig. 4 indicate that the RMS of
Horizontal Position Error was $42.68 \mathrm{~m}$ when using only the satellite system. However G3V1 is $17.24 \mathrm{~m}, \mathrm{G} 3 \mathrm{~V} 2$ is $15.16 \mathrm{~m}$, $\mathrm{G} 3 \mathrm{~V} 3$ is $11.13 \mathrm{~m}, \mathrm{G} 4 \mathrm{~V} 1$ is $12.04 \mathrm{~m}, \mathrm{G} 4 \mathrm{~V} 2$ is $11.26 \mathrm{~m}$, and G4V3 is $8.72 \mathrm{~m}$. The performance of Horizontal Position Error was improved by $59.60 \%, 64.47 \%, 73.92 \%, 71.79 \%$, $73.61 \%$, and 79.56 .

The experimental results of Fig. 6 indicate that the RMS of Horizontal Position Error was $45.64 \mathrm{~m}$ when using only the satellite system. However G3V1 is $20.32 \mathrm{~m}, \mathrm{G} 3 \mathrm{~V} 2$ is $16.68 \mathrm{~m}$, $\mathrm{G} 3 \mathrm{~V} 3$ is $12.75 \mathrm{~m}, \mathrm{G} 4 \mathrm{~V} 1$ is $13.24 \mathrm{~m}, \mathrm{G} 4 \mathrm{~V} 2$ is $11.49 \mathrm{~m}$, and G4V3 is $10.24 \mathrm{~m}$. The performance of the Horizontal Position Error was improved by $55.47 \%, 63.45 \%, 72.06 \%, 70.99 \%$, $74.82 \%$, and $77.56 \%$.

The experimental results of Fig. 7 indicate that the RMS of Horizontal Position Error was $49.82 \mathrm{~m}$ when using only the satellite system. However G3V1 is $22.84 \mathrm{~m}, \mathrm{G} 3 \mathrm{~V} 2$ is $16.07 \mathrm{~m}$, $\mathrm{G} 3 \mathrm{~V} 3$ is $11.70 \mathrm{~m}, \mathrm{G} 4 \mathrm{~V} 1$ is $12.46 \mathrm{~m}, \mathrm{G} 4 \mathrm{~V} 2$ is $11.95 \mathrm{~m}$, and G4V3 is $9.56 \mathrm{~m}$. The performance of the Horizontal Position Error was improved by $54.15 \%, 67.74 \%, 76.51 \%, 74.98 \%$, $76.01 \%$, and $80.81 \%$.

The experimental results of Fig. 9 indicate that the RMS of Horizontal Position Error was $159.27 \mathrm{~m}$ when using only the satellite system. However G3V1 is $7.57 \mathrm{~m}, \mathrm{G} 3 \mathrm{~V} 2$ is $7.37 \mathrm{~m}$, G3V3 is $7.29 \mathrm{~m}, \mathrm{G} 4 \mathrm{~V} 1$ is $7.24 \mathrm{~m}, \mathrm{G} 4 \mathrm{~V} 2$ is $7.13 \mathrm{~m}$, and G4V3 is $7.05 \mathrm{~m}$. The performance of the Horizontal Position Error was improved by $95.24 \%, 95.37 \%, 95.42 \%, 95.45 \%, 95.52 \%$, and $95.57 \%$.

The experimental results of Fig. 10 indicate that the RMS of Horizontal Position Error was $125.57 \mathrm{~m}$ when using only the satellite system. However G3V1 is $7.82 \mathrm{~m}, \mathrm{G} 3 \mathrm{~V} 2$ is $7.25 \mathrm{~m}, \mathrm{G} 3 \mathrm{~V} 3$ is $7.31 \mathrm{~m}, \mathrm{G} 4 \mathrm{~V} 1$ is $7.48 \mathrm{~m}, \mathrm{G} 4 \mathrm{~V} 2$ is $7.22 \mathrm{~m}$, and G4V3 is $7.03 \mathrm{~m}$. The performance of the Horizontal Position Error was improved by $93.77 \%, 94.22 \%, 94.17 \%, 94.04 \%$, $94.25 \%$, and $94.40 \%$.

The experimental result simulation shown in Fig. 12 indicates that the RMS of Horizontal Position Error was $66.09 \mathrm{~m}$ when using only the satellite system. However G3V1 is $7.40 \mathrm{~m}, \mathrm{G} 3 \mathrm{~V} 2$ is $7.42 \mathrm{~m}, \mathrm{G} 3 \mathrm{~V} 3$ is $7.28 \mathrm{~m}, \mathrm{G} 4 \mathrm{~V} 1$ is $7.38 \mathrm{~m}$, G4V2 is $7.32 \mathrm{~m}$, and G4V3 is $7.11 \mathrm{~m}$. The performance of the Horizontal Position Error was improved by $88.80 \%, 88.76 \%$, $88.98 \%, 88.83 \%, 88.92 \%$, and $89.24 \%$.

The experimental result simulation shown in Fig. 13 indicates that the RMS of Horizontal Position Error was $66.15 \mathrm{~m}$ when using only the satellite system. However G3V1 is $7.72 \mathrm{~m}, \mathrm{G} 3 \mathrm{~V} 2$ is $7.54 \mathrm{~m}, \mathrm{G} 3 \mathrm{~V} 3$ is $7.36 \mathrm{~m}, \mathrm{G} 4 \mathrm{~V} 1$ is $7.53 \mathrm{~m}$, G4V2 is $7.37 \mathrm{~m}$, and G4V3 is $7.33 \mathrm{~m}$. The performance of the Horizontal Position Error was improved by $88.33 \%, 88.60 \%$, $88.87 \%, 88.61 \%, 88.86 \%$, and $88.92 \%$.

\section{ACKNOWLEDGMENT}

This work was supported by the DGIST R\&D Program of the Ministry of Science, ICT and Technology of Korea(15-IT-01).

\section{REFERENCES}

[1] J. B. Tsui, Fundamentals of Global System Receivers, A Software Approach, John Wiley \& Sons, 2000, pp. 10-27.

[2] P. Ramjee and R. Marina, Applied Satellite Navigation Using GPS, Galileo, and Augmentation Systems, Artech House, 2005.

[3] W. B. Hofmann, H. Lichtenegger, and J. Collins, GPS Fifth, 2001. 
[4] D. K. Elliott, Understanding GPS: Principles and Applications, Artech House, 1996.

[5] W. Jinling, G. Matthew, L. Andrew, J. W. Jack, H. Songlai, and S. David, "Intergration of GPS/INS/vision sensors to navigate unmanned aerial vehicles," The International Archives of the Photogrammetry, Reomote Sensing and Spatial information Sciences, vol. 37, pp. 963-969, Beijing, 2008.

[6] S. Godha and M. E. Cannon, "Integration of DGPS with a low cost MEMS-based Inerial Measurement Unit(IMU) for land vehicle navigation application," in Proc. ION GPA-05, Institute of navigation, Long Beach, 2005, pp. 333-345.

[7] A. Broggi, C. Caraffi, R. I. Fedriga, and P. Grisleri, "Obstacle detection with stereo vision for off-road vehicle navigation," in Proc. IEEE Computer Society Conference on Computer Vision and Pattern Recognition, 2005, vol. 3, pp. 65-72.

[8] Y. C. Lim, M. H. Lee, C. H. Lee, S. Kwon, and J. H. Lee, "Improvement of stereo vision-based position and velocity estimation and tracking using a strip-based disparity estimation and inverse perspective map-based extended kalman filter," Optics and Lasers in Engineering, vol. 48, no. 9, pp. 859-868, 2010.

[9] M. Bertozzi, A. Broggi, M. Cellario, A. Fascoli, P. Lombardi, and M. Porta, "Artificial vision in road vehicles," Proceeding of the IEEE, vol. 90, no. 7, pp. 1258-1271, 2002.

[10] N. Srinivasa, "A vision-based vehicle detection and tracking method for forward collision warning," in Proc. IEEE Intelligent Vehicle Symposium, 2002, pp. 626-631.
[11] V. Lemonde and M. Devy, "Obstacle detection with stereo vision," Mechatronics and Robotics, Germany, 2004.

[12] E. J. Rossetter, J. P. Switkes, and J. C. Gerfes, "Experimental validation of the potential field lanekeeping system," International Journal of Automotive Technology, vol. 5, no. 95, 2004.

[13] R. Havard and H. Leonhard, Gaussian Markov Random Fields, 2007.

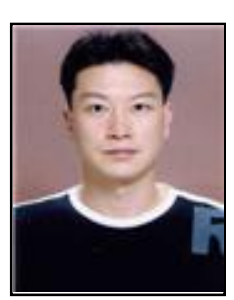

Chi-Ho Park received $\mathrm{PhD}$ and master degrees in electronic communication engineering from Kwangwoon University, Korea in 2001 and 2008. He is currently studying for the positioning for unmanned vehicles in DGIST (Daegu Gyeongbuk Institute of Science \& Technology).

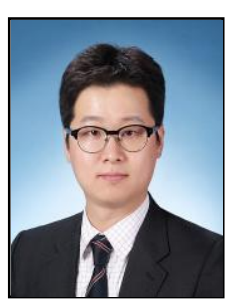

Nam-Hyeok Kim received the master degree in spatial information engineering from Kyungpook National University, Korea in 2014. He received a bachelor degree in spatial information engineering from University of Seoul, Korea in 2009. He is currently studying for the positioning for unmanned vehicles in DGIST (Daegu Gyeongbuk Institute of Science \& Technology) and on $\mathrm{PhD}$ course in Kyungpook National University. His research interests includes electronic map for unmanned vehicles. 